\title{
A Rare Case of Celiac Presenting at an Atypical Age with Multisystem Involvement
}

\author{
Uttayan Chakrabarti ${ }^{1, *}$, Manisha Bais Thakur ${ }^{1}$, Geetika Khanna ${ }^{2}$ \\ ${ }^{1}$ Department of Medicine, Vardhman Mahavir Medical College \& Safdarjung Hospital, New Delhi, India \\ ${ }^{2}$ Department of Pathology, Vardhman Mahavir Medical College \& Safdarjung Hospital, New Delhi, India \\ *Corresponding author: uttayanchakrabarti@gmail.com
}

Received September 10, 2019; Revised October 21, 2019; Accepted December 04, 2019

\begin{abstract}
Celiac Disease is an autoimmune disorder due to hypersensitivity to gluten. Gluten free diet usually reverses the pathological changes but in 7-30 \% of cases when there is no reversal one should look for the evidence of Microscopic colitis. Celiac disease is also associated with multiple haematological and renal abnormalities and here we report an atypical case of Celiac presenting in an unusual age with multisystem involvement.
\end{abstract}

Keywords: gluten free diet, microscopic colitis, multi-system involvement

Cite This Article: Uttayan Chakrabarti, Manisha Bais Thakur, and Geetika Khanna, "A Rare Case of Celiac Presenting at an Atypical Age with Multisystem Involvement.” International Journal of Celiac Disease, vol. 7, no. 3 (2019): 86-89. doi: 10.12691/ijcd-7-3-4.

\section{Introduction}

Celiac disease is an autoimmune disorder characterized by intolerance of gluten, the storage protein of wheat and similar grains. Exposure to gluten leads to damage of the small bowel mucosa characterized by intraepithelial lymphocytosis, crypt hypertrophy, and varying degrees of villous atrophy with resolution of the pathological changes on withdrawal of gluten from the diet. [1] But in 7-30\% of individuals there have been persistent symptoms despite being compliant to the diet. The diet are, however, common, estimated to occur in $7 \%$ to $30 \%$ of individuals with celiac disease. Microscopic colitis has been shown in various studies among the leading causes of persistent or recurrent diarrhea in patients with celiac disease, despite adherence to the diet. [2] Although the simultaneous diagnosis of both microscopic colitis and celiac disease should be undertaken with caution because intraepithelial lymphocytosis in duodenal biopsies, in the absence of villous atrophy, has been suggested in patients with microscopic colitis as well. [3] Celiac disease may not have the classical symptoms in which they have atypical presentations and are found to have multiple haematological and renal abnormalities as well. We report an atypical case of Celiac which presented at a late age and was found to have multisystem involvement.

\section{Case Report}

A 50 year old non diabetic, non hypertensive,non alcoholic male presented with 6 months history of 6-7 episodes of painless, copious, non-bloody loose stools with 5 months history of bilateral lower limb swelling without any vomiting, fever, joint pain, rashes or decreased urine output. He was investigated to have features of malabsorption syndrome with iron deficiency anaemia, hypoproteinemia, hypocalcemia, a low vitamin D3 along with thrombocytopenia with a normal urine examination. An upper GI endoscopy along with biopsy and serology were compatible with Celiac disease(Marsh Grade2) [Figure 1, Figure 2], was put on gluten free diet following which only stool frequency decreased. He was readmitted after 2 months, inspite of being compliant to gluten free diet, again with 8-10 episodes of loose stools, bilateral pedal edema, with fever and decreased urine output since 7 days.

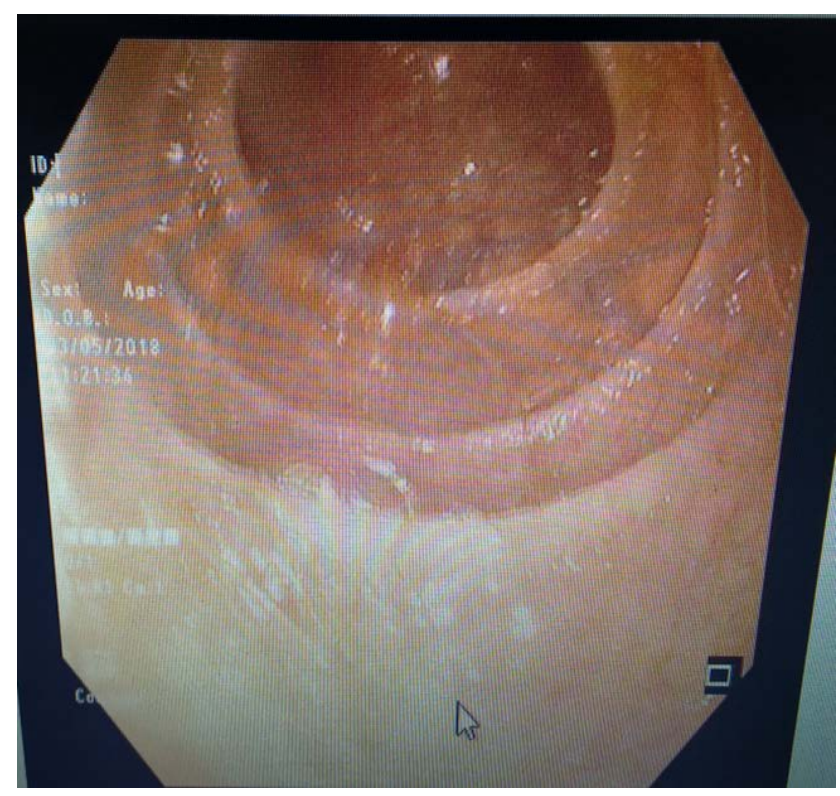

Figure 1. Upper GI endoscopy showing flattening of D2 folds 


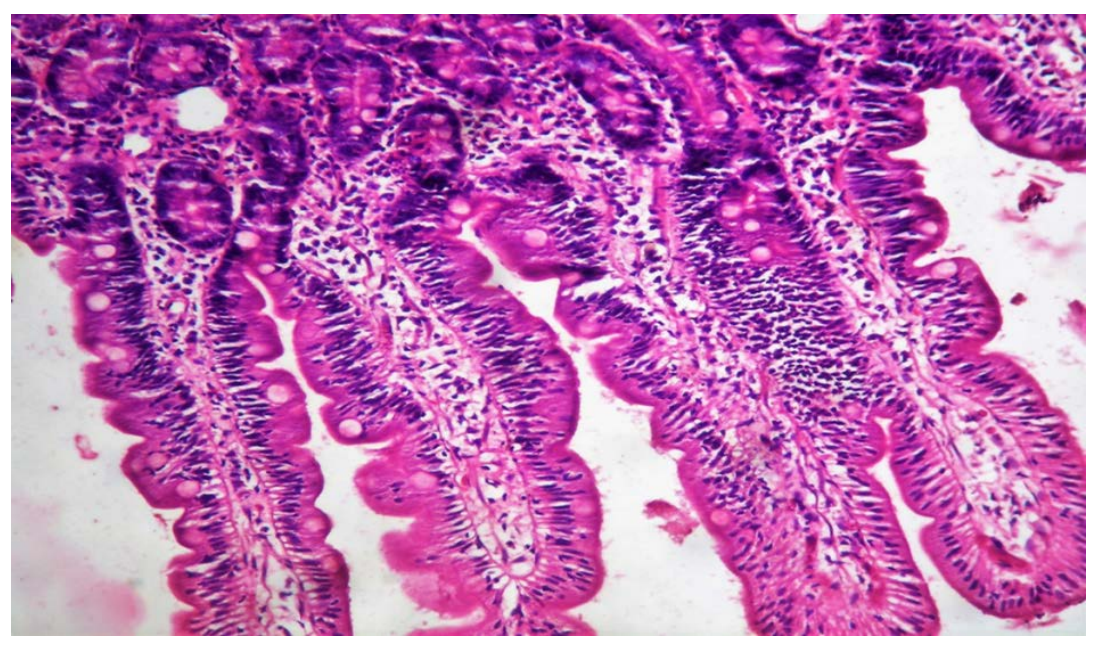

Figure 2. Duodenal biopsy showing almost normal villus architecture with moderate degree of lymphoplasmacytic Infiltrate in the lamina propria, intraepithelial lymphocytes with crypt hyperplasia s/o Celiac disease (Marsh Grade2)

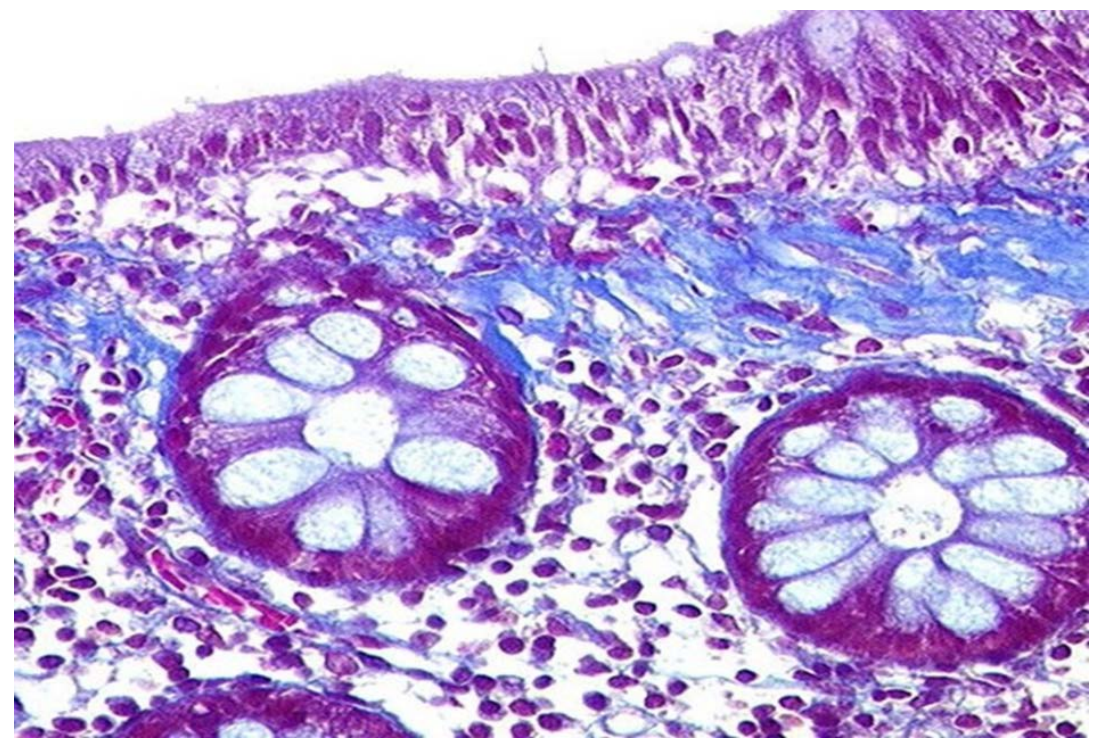

Figure 3. Sigmoid biopsy showing irregular subepithelial collagenisation (Masson’s trichrome stain) extending into the lamina propria s/o Collagenous colitis.No crypt abscesses, cryptitis and ulceration.

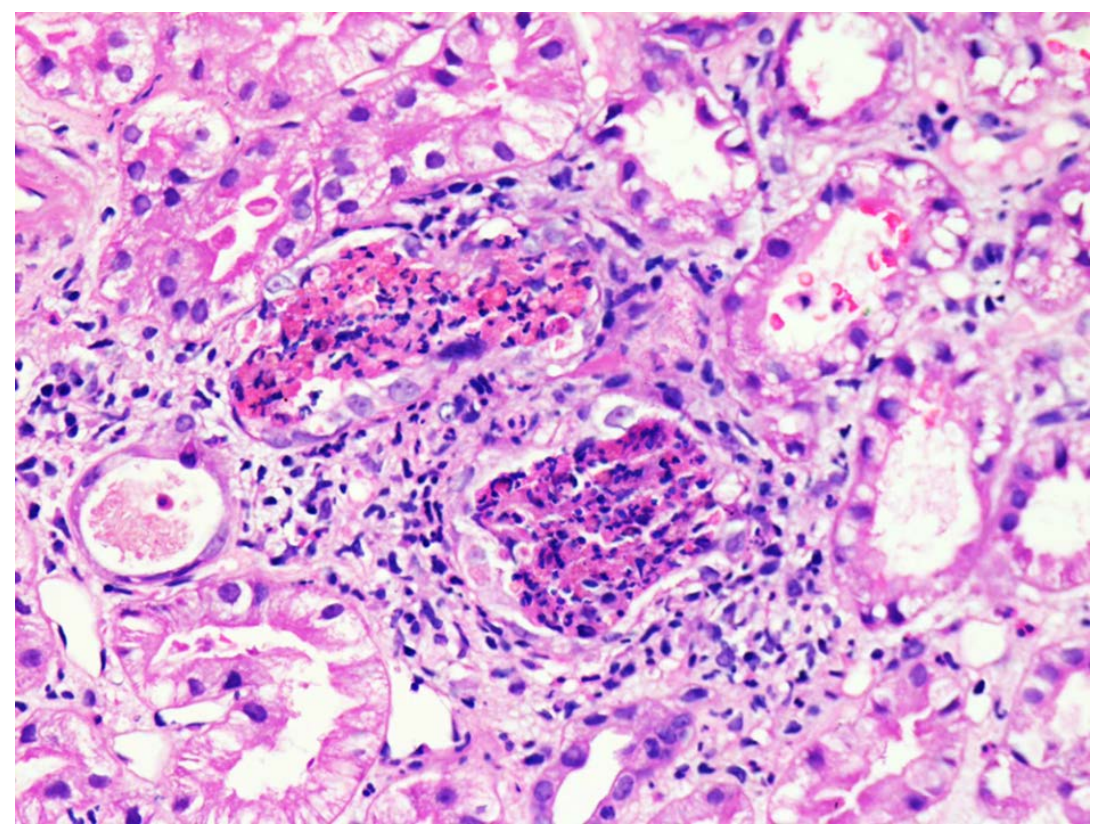

Figure 4. Kidney biopsy showing mesangial proliferation and tubules showing acute \& chronic inflammatory infiltrate in the lining epithelium, interstitium indicating tubular injury 


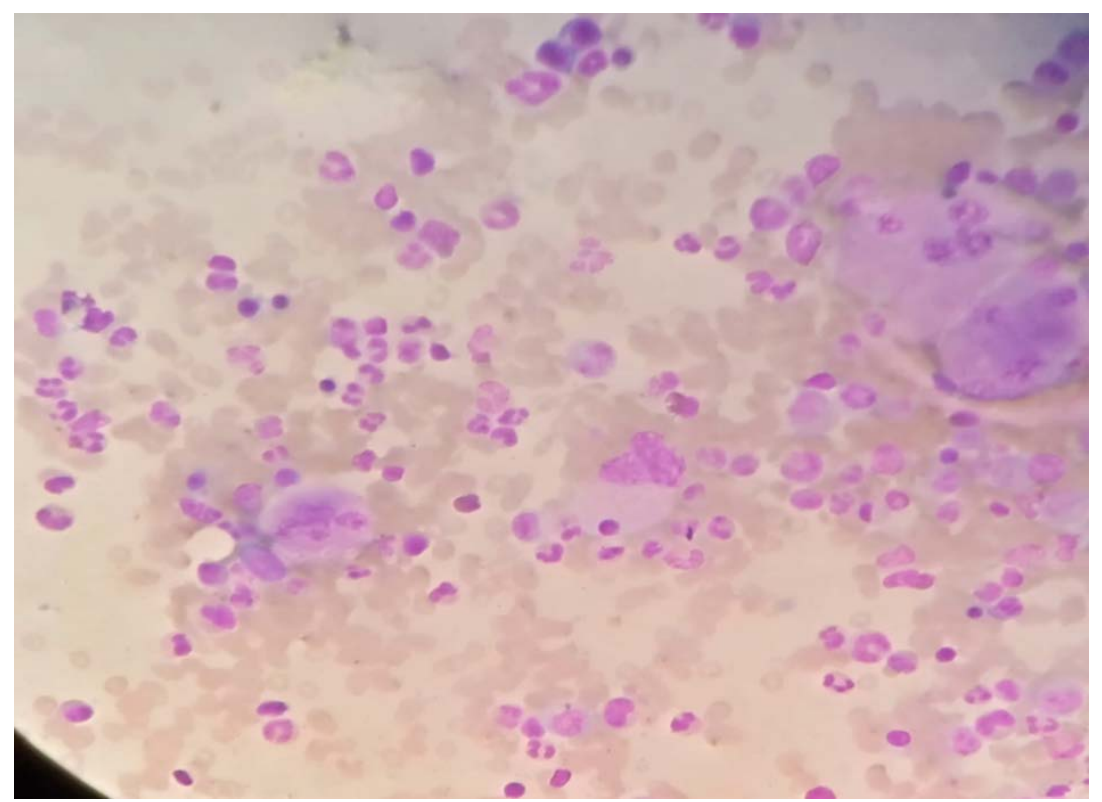

Figure 5. Bone marrow biopsy suggestive of normocellular bone marrow with increase in the number of megakaryocytes some of which showed abnormal morphology s/o ITP

On Examination, he had pallor, pedal Edema, scaly plaques over lower limbs, P/A examination revealed as cites. Chest, CVS, CNS examinations were within normal limits.

Investigations revealed $\mathrm{Hb}-7.4 \mathrm{~g} / \mathrm{dl}$, TLC-34,800/ $\mathrm{l}(\mathrm{N}-$

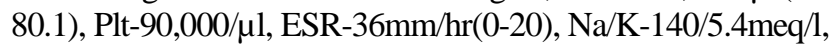
U/Cr-92/4.1mg/dl, Urine-Albumin 4+, Sugar -nil, 1-2 Pus cells, 30-40 RBC/hpf, 1-2 epithelial cells, Dysmorphic RBCs+, 24 hour urine protein-1.8g/dl, Urine \& Blood C/S- no growth.

C3-43.33mg/dl(90-180), ASO, IgA, C4-WNL, ANA, Anti ds DNA, p-ANCA, c-ANCA-negative, USG W/A Increased bilateral renal echotexture with gross ascites. Colonoscopy revealed diminished vascular pattern in rectum, rectosigmoid, biopsy suggestive of Collagenous microscopic colitis (Figure 3).

A kidney biopsy was done which was suggestive of Mesangioproliferative Glomerulonephritis with Acute kidney injury (Figure 4) while bone Marrow Biopsy revealed normocellular marrow with increase in the number of megakaryocytes some of which showed abnormal morphology suggestive of Immune Thrombocytopenic purpura (Figure 5). So a final Diagnosis of Celiac disease with Collagenous Microscopic colitis with Mesangioproliferative Glomerulonephritis with ITP was established.

Patient was initiated on steroids to which the response was dramatic with resolution of all his symptoms. On gradual tapering doses of steroids after 3 months of follow up there was resolution of all biochemical and haematological parameters.

\section{Discussion}

Celiac disease is associated with a 50 to 70 times greater risk of microscopic colitis which should be ruled out in patients of celiac disease with persistent or recurrent diarrhea inspite of being adherent to the diet. [2] Collagenous colitis is characterized by the presence of a subepithelial collagen band at least $20 \mu \mathrm{m}$ thick and the proposed mechanisms of chronic diarrhea being reduced net absorption of sodium and chloride, active secretion of chloride and barrier of water reabsorption due to collagen band. [4] Anemia is a frequent finding in Celiac and often the presenting feature which primarily results from impaired absorption of iron and requires a gluten free diet and iron supplementation until the iron stores have been restored. This process can take as long as a year for the hemoglobin to normalize and 2 years for the iron stores to be replete. [5] Native immune system is important in the pathogenesis of Celiac disease and toll-like receptors (TLRs) also play a key role.In Celiac disease, tTG antibodies induce TLR4 activation and the TLR4 expression in platelets leads to thrombocytopenia. [6] Platelet derived growth factor (PDGF) is a potent mitogen for cells of mesenchymal origin including smooth muscle cells and glial cells. It has been reported that PDFG levels are elevated in patients with ITP. PDGF increases cell proliferation which explains the mesangial proliferation. [7]

\section{Conclusion}

Microscopic colitis should always be ruled out by colonoscopy in cases of non responders to gluten free diet and biopsy should be taken from a normal looking mucosa for arriving at a diagnosis. It should be kept in mind that Celiac is associated with multiple systemic abnormalities which should be sought as well and treating the root cause always cures the patient. This was a rare case of Celiac which presented at an older age, had associated microscopic colitis and had haematological and renal abnormalities secondary to Celiac disease.

\section{References}

[1] Green PH, Cellier C. Celiac disease. N Engl J Med 2007; 357: 1731-1743. 
[2] Fine KD, Meyer RL, Lee EL. The prevalence and causes of chronic diarrhea in patients with celiac sprue treated with a glutenfree diet. Gastroenterology 1997; 112: 1830-1838.

[3] Brown I, Mino-Kenudson M, Deshpande V, et al. Intraepithelial lymphocytosis in architecturally preserved proximal small intestinal mucosa: an increasing diagnostic problem with a wide differential diagnosis. Arch Pathol Lab Med 2006; 130: 1020-1025.

[4] Bürgel N., Bojarski C., Mankertz J., Zeitz M., Fromm M., Schulzke J. Mechanisms of diarrhea in collagenous colitis. Gastroenterology. 2002; 123(2): 433-443.
[5] De Vizia B, Poggi V, Conenna R, Fiorillo A, Scippa L. Iron absorption and iron deficiency in infants and children with gastrointestinal diseases. J Pediatr Gastroenterol Nutr. 1992; 14: 21-26.

[6] Aslam R, Speck ER, Kim M, Crow AR, Bang KW, Nestel FP, et al. Platelet Toll-like receptor expression modulates lipopolysaccharide-induced thrombocytopenia and tumor necrosis factor-alpha production in vivo. Blood. 2006; 107: 637-41.

[7] Katoh O, Kimura A, Fujimura K, Kuramoto A. Progression activity for platelet-derived growth factor in plasma of patients with idiopathic thrombocytopenic purpura and aplastic anemia. Nihon Ketsueki Gakkai Zasshi. 1989; 52(1): 113-117.

(C) The Author(s) 2019. This article is an open access article distributed under the terms and conditions of the Creative Commons Attribution (CC BY) license (http://creativecommons.org/licenses/by/4.0/). 